\title{
Synthesis and antimicrobial activity of coumarinyl Schiff base derivatives
}

\author{
Arun Mishra, Jaynt Rathod, Dipti Namera, Shailesh Thakrar, Anamik Shah* \\ Chemical Research Laboratory, Department of Chemistry, Saurashtra University, \\ Rajkot - 360005, India \\ *E-mail address: anamik_shah@hotmail.com
}

\begin{abstract}
We have reported some novel N'-[(1)-ethylidene]-2-(6-methyl-2-oxo-2H-chromen-4-yl) acetohydrazide synthesized by conventional method. The reaction of 2-(6-methyl-2-oxo-2H-chromen4-yl)acetohydrazide with substituted benzaldehyde in methanol as a solvent yielded a series of (7a-1). The structures of all synthesized compounds are well characterized by Mass, FT-IR, ${ }^{1} \mathrm{H}$ NMR, ${ }^{13} \mathrm{C}$ NMR and elemental analysis. Moreover, all synthesized compounds were screened for in vitro antimicrobial activity against the gram positive (Staphylococcus aureus, Bacilluas subtilis) and gram negative (Escherichia coli, P. aeruginosa, K. pneunonae, Enterobacter) bacterial strain. In which some the compounds show potential inhibition against the test organisms.
\end{abstract}

Keywords: gram positive bacterial; gram negative bacterial; derivatives of coumarin

\section{INTRODUCTION}

Coumarin and its derivatives represent one of the most active classes of compound possessing a wide spectrum of biological activity [1-4]. The synthesis of coumarin derivatives has attracted considerable attention of organic and medicinal chemists as these are widely used as therapeutic agents. Coumarin-containing antibiotics such as novobiocin (I) amino coumarin core produced by Streptomyces species, have gained renewed interest since the discovery that they are potent inhibitors of bacterial DNA gyrase, which is essential for cell viability [5,6]. In addition, these coumarin antibiotics are potent against methicilin resistant strains of Staphylococci species, currently one of the major concerns in the treatment of bacterial infections [7]. Effective reversal of breast cancer resistant protein (BCRP)-mediated drug resistance by novobiocin was studied by Shozawa et al.[8] and Nakatomi et al. [9].

This has renewed interest in coumarin based compounds. Alternatively Schiff bases are also important intermediates for the synthesis of some bioactive compounds such as $\beta$ lactams [10-13]. Furthermore, they are reported to show a variety of interesting biological actions, including antibacterial [14-16], antifungal [17,18] anti mouse hepatitis virus (MHV) [19], inhibition of herpes simplex virus type 1 (HSV-1) and adenovirus type 5 (Ad 5) [20], anticancer [21-24], antimosquito larvae [25] and herbicidal activities [26]. The hydrazides attatched to heterocycles like pyridine, pyrazines is believed to be wonderful chemotherapeutic agents. The isoniazide and pyrazinamide are classical examples of such 
drugs used as antitubercular agents. In the same vein, the coumarin scaffold when appended with $-\mathrm{CH}_{2}-\mathrm{C}=\mathrm{O}$ - linker gave very good antiviral properties.

On the basis of these findings, in the present work, it was planned to club coumarin moiety with a spacer $-\mathrm{CH}_{2}-\mathrm{C}=\mathrm{O}$ - with an aim to prepare compounds which mimick "hydrazide" like properties. In the present work, coumarin-4-acetic acids were prepared by literature method \& the acids were further converted into respective esters, which on treatment with hydrazine hydrate afforded to give 22 new compounds. In the present work 6methyl coumarin-4-acetic acid (III) was prepared by Pechmann condensation of resorcinol and citric acid using sulfuric acid as condensing agent. The compounds were prepared by the hydrazide with appropriately reacting aldehydes to obtain N'-[(1)-ethylidene]-2-(6-methyl-2oxo-2H-chromen-4-yl)acetohydrazide in good yields.

\section{EXPERIMENTALS}

\section{1. Material and method}

Melting points were determined by open capillary tubing and are uncorrected. Purification of the compounds was routinely checked by TLC on silica gel-G plates of $0.5 \mathrm{~mm}$ thickness and spots were located by iodine and UV. Mass spectra were recorded on Shimadzu GCMS-QP-2010 model using Direct Injection Probe technique. The elemental analysis for C, $\mathrm{H}$ and N was done on a Perkin-Elmer Analyzer 2440. Infrared spectra $\left(v m a x-\mathrm{cm}^{-1}\right)$ were recorded on a Beckmann FT-IR 1800, using KBr pallet method. ${ }^{1} \mathrm{H}$ NMR spectra were recorded on Bruker Advance $400 \mathrm{MHz}$ spectrometer in DMSO- $\mathrm{d}_{6}$ by using TMS as a standard Chemical shifts (d) are given in ppm relative to TMS. All the chemicals and solvents were purchased from Spectrochem, S D Fine Chemicals and Loba Chemie (India) and used without further purification.

\section{Preparation of (6-metheyl-2-oxo-2H-chromen-4-yl)acetic acid}

Citric acid $(0.1 \mathrm{M})$ and concentrated sulfuric acid $(32 \mathrm{ml})$ were mixed in a RBF and shaken for half an hour. The mixture was then slowly warmed on a water bath up to $70{ }^{\circ} \mathrm{C}$ temperature. The mixture was left at this temperature for 10-15 minutes and as soon as gas slackened, the flask was removed from the bath allowed to stand for 15 minutes, till the liquid becomes clear, free from gas bubbles and then cooled to $10{ }^{\circ} \mathrm{C}$ in ice bath. p-Cresol $(0.1 \mathrm{M})$ and concentrated sulfuric acid $(14 \mathrm{ml})$ were added gradually to the solution and the mixture was shaken, taking care that the temperature doesn't rise above $10^{\circ} \mathrm{C}$. The dark color solution was left for 48 hour at room temperature. It was then poured into ice-cold water, when a bulky solid separated. It was filtered and treated with sodium bicarbonate $(10 \%)$ solution and then insoluble matter was removed by filtration. The filtrate, on acidification gave (6-methyl2-oxo-2Hchromen-4-yl)acetic acid. The purity of the compound was checked by TLC (Acetone: Hexane: 5:5), M.P. $185-187^{\circ} \mathrm{C}$ (M.P. Reported $\left.=181{ }^{\circ} \mathrm{C} 73\right)\left\{\mathrm{R}_{\mathrm{f}}\right.$ Value: 0.54$\}$.

\section{Preparation of ethyl (6-methyl-2-oxo-2H-chromen-4-yl)acetate}

(6-methyl-2-oxo-2H-chromen-4-yl)acetic acid (10 gm) was taken into $250 \mathrm{ml} \mathrm{RBF}$ and to this $70 \mathrm{ml}$ ethanol along with few drops of sulfuric acid was added and this reaction mixture was allowed to reflux on water bath for $4 \mathrm{hr}$ and the reaction was monitored with TLC (Ethyl Acetate : Hexane: 4:6). The reaction mixture was poured into ice-water to obtain the product which was filtered and washed with distilled water. M.P. $136^{\circ} \mathrm{C}$. 


\section{Preparation of 2-(6-methyl-2-oxo-2H-chromen-4-yl)acetohydrazide}

A mixture of ethyl (6-methyl-2-oxo-2H-chromen-4-yl)acetate (10 gm) and hydrazine hydrate $(98 \% 20 \mathrm{ml})$ was allowed to reflux for $8 \mathrm{hr}$ in the heating metal. The completion of reaction was observed by TLC. The reactio mixture was poured in ice, filtered and crystallised from alcohol. M.P. $>300^{\circ} \mathrm{C}$.

\section{Preparation of $N^{\prime}$-[(1)-ethylidene]-2-(6-methyl-2-oxo-2H-chromen-4-yl)acetohydrazide}

A mixture of 2-(6-methyl-2-oxo-2H-chromen-4-yl)acetohydrazide $(0.01 \mathrm{~mol})$ and aldehyde $(0.01 \mathrm{~mol})$ was refluxed in absolute alcohol for $12 \mathrm{hrs}$. The reaction mixture was cooled to room temperature and was poured to ice and filtered the product. The product was recrystallised from alcohol.

\section{REACTION SCHEME}<smiles>O=C(O)CC(O)(CC(=O)O)C(=O)O</smiles>

citric acid

1

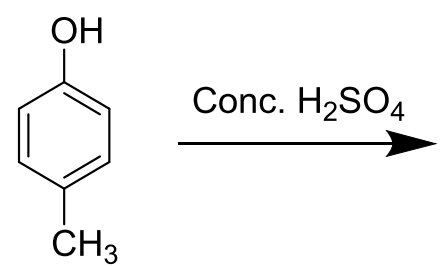

2<smiles>Cc1ccc2oc(=O)cc(CC(=O)O)c2c1</smiles>
Reflux $\begin{aligned} & \text { Conc. } \mathrm{H}_{2} \mathrm{SO}_{4} \\ & \mathrm{CH}_{3} \mathrm{CH}_{2} \mathrm{OH}\end{aligned}$<smiles>[R]OC(C)N</smiles>

5<smiles>CCOC(=O)Cc1cc(=O)oc2ccc(C)cc12</smiles>

4 
<smiles>Cc1ccc2oc(=O)cc(CC(=O)NN)c2c1</smiles>

5<smiles>O=Cc1ccccc1</smiles>

6<smiles>[R]Oc1ccccc1/C=N/NC(=O)Cc1cc(=O)oc2ccc(C)cc12</smiles>

7a-I

\section{SPECTRAL DATA}

N'-benzylidene-2-(6-methyl-2-oxo-2H-chromen-4-yl)acetohydrazide (7a)

MP: $210-212^{\circ} \mathrm{C}$; IR $\left(\mathrm{cm}^{-1}\right): 3423.4,2923.9,2853.5,1718.4,1654.2,1425.3,1361.7,804.3$, 759.9, ${ }^{1} \mathrm{H}$ NMR (DMSO-d6), $\delta$ ppm: 2.26 (s, 3H), 3.52 (s, 2H), 5.85 (s, 1H), 6.67-6.70 (q, 2H), $7.25(\mathrm{~d}, 1 \mathrm{H}), 7.39-7.46(\mathrm{~m}, 3 \mathrm{H}), 7.78-7.91(\mathrm{~m}, 3 \mathrm{H}), 9.55(\mathrm{~s}, 1 \mathrm{H})$.

N'-(4-methoxybenzylidene)-2-(6-methyl-2-oxo-2H-chromen-4-yl)acetohydrazide (7b) MP: $150-152{ }^{\circ} \mathrm{C}$; IR $\left(\mathrm{cm}^{-1}\right)$ : $3421.2,2859.6,1715.4,1654.5,1425.3,1365.1,760.2,{ }^{1} \mathrm{H}$ NMR (DMSO-d6), $\delta$ ppm: $2.30(\mathrm{~s}, 1 \mathrm{H}), 2.82(\mathrm{~s}, 2 \mathrm{H}), 3.87(\mathrm{~s}, 3 \mathrm{H}), 6.05(\mathrm{~s}, 1 \mathrm{H}), 7.06-7.08$ (d, 2H, $8 \mathrm{~Hz}), 7.24-7.28(\mathrm{~m}, 2 \mathrm{H}), 7.48(\mathrm{~s}, 1 \mathrm{H}), 7.78-7.80(\mathrm{~d}, 2 \mathrm{H}), 6.27(\mathrm{~s}, 1 \mathrm{H}), 8.18(\mathrm{~s}, 1 \mathrm{H})$.

N'-(4-fluorobenzylidene)-2-(6-methyl-2-oxo-2H-chromen-4-yl)acetohydrazide (7c)

MP: $168-170{ }^{\circ} \mathrm{C}$; IR $\left(\mathrm{cm}^{-1}\right)$ : 3342.4, 3263.3, 3039.6, 2854.5, 1706.9, 1664.5, 1541.0, 1506.3, 1431.1, 777.3, 756.0, ${ }^{1} \mathrm{H}$ NMR (DMSO-d6), $\delta$ ppm: $2.28(\mathrm{~s}, 3 \mathrm{H}), 2.75(\mathrm{~s}, 2 \mathrm{H}), 6.12(\mathrm{~s}, 1 \mathrm{H})$, 7.24-7.28 (d, 2H), 7.29-7.31 (d, 2H, J-8Hz), 7.37 (s, 1H), 7.61-7.63 (t, 2H), $6.28(, 1 \mathrm{H})$, $8.11(\mathrm{~s}, 1 \mathrm{H})$.

N'-(4-hydroxybenzylidene)-2-(6-methyl-2-oxo-2H-chromen-4-yl)acetohydrazide (7d) MP: $230-232{ }^{\circ} \mathrm{C}$; IR $\left(\mathrm{cm}^{-1}\right)$ : 3345.2, 3270.2, 3031.5, 2900.3, 1705.4, 1664.2, 1506.3, 1421.3, 817.8, 756.0, ${ }^{1} \mathrm{H}$ NMR (DMSO-d6), $\delta$ ppm: 2.27 (s, 3H), 2.70 (s, 2H), 5.30 (s, 1H), 6.12 (s, $1 \mathrm{H}), 6.78(\mathrm{~d}, 2 \mathrm{H}), 7.21(\mathrm{~d}, 1 \mathrm{H}), 7.24(\mathrm{~d}, 1 \mathrm{H}), 7.45(\mathrm{~s}, 1 \mathrm{H}), 7.65(\mathrm{~d}, 2 \mathrm{H}), 6.25(\mathrm{~s}, 1 \mathrm{H}), 8.09$ (s, $1 \mathrm{H})$.

2-(6-methyl-2-oxo-2H-chromen-4-yl)-N'-((E)-3-phenylallylidene)acetohydrazide (7e) MP: 226-228 ${ }^{\circ} \mathrm{C}$; IR $\left(\mathrm{cm}^{-1}\right)$ : 3368.4, 3021.2, 2885.1, 2260.2, 2106.2, 1706.2, 1659.3, 1525.3, 435.2, 1361.3, 1022.2, 804.3, 690.5, ${ }^{1} \mathrm{H}$ NMR (DMSO-d6), $\delta$ ppm: 2.21 (s, 3H), 2.71 (s, 2H), $5.96(\mathrm{~s}, 1 \mathrm{H}), 6.70-6.72(\mathrm{dd}, 1 \mathrm{H}), 5.27(\mathrm{~s}, 1 \mathrm{H}), 7.10-7.32(\mathrm{~m}, 6 \mathrm{H}), 7.21(\mathrm{~s}, 1 \mathrm{H}), 7.35-7.40(\mathrm{~m}$, $3 \mathrm{H})$.

\section{2-(6-methyl-2-oxo-2H-chromen-4-yl)-N'-(3,4,5-trimethoxybenzylidene)acetohydrazide} (7f)

MP: $208-210^{\circ} \mathrm{C}$; IR $\left(\mathrm{cm}^{-1}\right): 3352.3,3011.3,2886.3,1740.2,1681.2,1710.6,1609.1,1552.3$, 1462.3, 1330.4, 1191.9, 759.3, 696.3, ${ }^{1} \mathrm{H}$ NMR (DMSO-d6), $\delta$ ppm: 2.42 (s, 3H), 2.79 (s, $2 \mathrm{H}), 3.65(\mathrm{~s}, 9 \mathrm{H}), 6.08(\mathrm{~s}, 1 \mathrm{H}), 6.18(\mathrm{~s}, 1 \mathrm{H}), 6.79-6.86(\mathrm{~m}, 2 \mathrm{H}), 7.36-7.42(\mathrm{~m}, 3 \mathrm{H})$.

N'-(3,4-dimethoxybenzylidene)-2-(6-methyl-2-oxo-2H-chromen-4-yl)acetohydrazide (7g) MP: $148-150{ }^{\circ} \mathrm{C}$; IR $\left(\mathrm{cm}^{-1}\right)$ : 3354.3, 3056.3, 2971.3, 2853.1, 1714.2, 1709.1, 1542.3, 1452.3, 1352.3, 1206.3, 1193.1, 842.3,654.3, ${ }^{1} \mathrm{H}$ NMR (DMSO-d6), $\delta$ ppm: 2.30 (s, 3H), 2.72 (s, 2H), $3.70(\mathrm{~s}, 6 \mathrm{H}), 6.08(\mathrm{~s}, 1 \mathrm{H}), 7.10-7.18(\mathrm{~m}, 3 \mathrm{H}), 7.26-7.28(\mathrm{~m}, 3 \mathrm{H}), 7.48(\mathrm{~s}, 1 \mathrm{H})$. 
N'-(3-methoxybenzylidene)-2-(6-methyl-2-oxo-2H-chromen-4-yl)acetohydrazide (7h) MP: $218-220^{\circ} \mathrm{C}$; IR $\left(\mathrm{cm}^{-1}\right)$ : $3362.1,3019.3,2951.3,1714.3,1705.6,1435.6,1361.3,1213.1$, 841.3, 695.3, ${ }^{1} \mathrm{H}$ NMR (DMSO-d6), $\delta$ ppm: 2.40 (s, 3H), 2.82 (s, 2H), 3.82 (s, 3H), 6.04 $(\mathrm{s}, 1 \mathrm{H}), 6.12(\mathrm{~s}, 1 \mathrm{H}), 6.85-6.91(\mathrm{~m}, 4 \mathrm{H}), 7.15-7.22(\mathrm{~m}, 3 \mathrm{H})$.

N'-(3-chlorobenzylidene)-2-(6-methyl-2-oxo-2H-chromen-4-yl)acetohydrazide (7i) MP: $162-164{ }^{\circ} \mathrm{C}$; IR $\left(\mathrm{cm}^{-1}\right)$ : $3369.2,2889.3,1715.6,1710.2,1552.6,1462.3,759.9,529.1,{ }^{1} \mathrm{H}$ NMR (DMSO-d6), $\delta$ ppm: 2.26 (s, 3H), 3.42 (s , 2H), 6.16 (s, 1H), $6.20(\mathrm{~s}, 1 \mathrm{H}), 6.82-6.90$ $(\mathrm{m}, 4 \mathrm{H}), 7.12-7.28(\mathrm{~m}, 3 \mathrm{H}), 7.32(\mathrm{~s}, 1 \mathrm{H})$.

N'-(2-chlorobenzylidene)-2-(6-methyl-2-oxo-2H-chromen-4-yl)acetohydrazide (7j) MP: $250-252{ }^{\circ} \mathrm{C}$; IR $\left(\mathrm{cm}^{-1}\right)$ : 3334.7, 3055.0, 2858.3, 1710.7, 1664.5, 1552.6, 1431.1, 1373.2, 788.8, 744.5, ${ }^{1} \mathrm{H}$ NMR (DMSO-d6), $\delta$ ppm: 2.24 (s, 3H), 3.44 (s , 2H), 6.18 (s, 1H), 6.24 (s, 1H), 6.74-6.88 (m, 4H), 7.08-7.18 (m, 3H), $7.25(\mathrm{~s}, 1 \mathrm{H})$.

N'-(4-chlorobenzylidene)-2-(6-methyl-2-oxo-2H-chromen-4-yl)acetohydrazide (7k)

MP: $230-232{ }^{\circ} \mathrm{C}$; IR $\left(\mathrm{cm}^{-1}\right)$ : 3334.7, 3055.0, 2858.3, 1710.7, 1664.5, 1552.6, 1431.1, 1373.1, 778.8, 734.5, 569.7, ${ }^{1} \mathrm{H}$ NMR (DMSO-d6), $\delta$ ppm: 2.24 (s, 3H), 3.45 (s, 2H), 6.20 (s, 1H), $6.24(\mathrm{~s}, 1 \mathrm{H}), 6.79-6.90(\mathrm{~m}, 4 \mathrm{H}), 7.06-7.29(\mathrm{~m}, 3 \mathrm{H}), 7.41(\mathrm{~s}, 1 \mathrm{H})$.

2-(6-methyl-2-oxo-2H-chromen-4-yl)-N'-(3-nitrobenzylidene)acetohydrazide (7l) MP: $212-214^{\circ} \mathrm{C}$; IR $\left(\mathrm{cm}^{-1}\right)$ : 3342.1, 3241.2, 3050.1, 2857.2, 1706.9, 1665.3, 1543.2, 1512.6, 1463.2, 825.1, 742.1, ${ }^{1} \mathrm{H}$ NMR (DMSO-d6), $\delta$ ppm: 2.28 (s, 3H), 3.21 (s, 2H), 6.35 (s, 1H), 7.24-7.28 (dd, 2H), $7.41(\mathrm{~s}, 1 \mathrm{H}), 7.18(\mathrm{t}, 1 \mathrm{H}), 8.70-8.83(\mathrm{~m}, 4 \mathrm{H}), 5.36(\mathrm{~s}, 1 \mathrm{H})$.

\section{PHYSICAL DATA TABLE}

Table 1

\begin{tabular}{|c|c|c|c|c|c|}
\hline Code & $\begin{array}{c}\text { Substitution } \\
\mathbf{R}\end{array}$ & $\begin{array}{c}\text { Substitution } \\
\mathbf{R}_{\mathbf{1}}\end{array}$ & $\begin{array}{c}\text { Molecular } \\
\text { formula }\end{array}$ & $\begin{array}{c}\mathbf{R}_{\mathbf{f}} \\
\text { Value }\end{array}$ & $\begin{array}{c}\text { M.P } \\
{ }^{\circ} \mathbf{C}\end{array}$ \\
\hline $\mathbf{7 a}$ & $6-\mathrm{CH}_{3}$ & $\mathrm{C}_{6} \mathrm{H}_{5}$ & $\mathrm{C}_{18} \mathrm{H}_{14} \mathrm{~N}_{2} \mathrm{O}_{3}$ & 0.32 & $210-212$ \\
\hline $\mathbf{7 b}$ & $6-\mathrm{CH}_{3}$ & $4-\mathrm{OCH}_{3}-\mathrm{C}_{6} \mathrm{H}_{4}$ & $\mathrm{C}_{19} \mathrm{H}_{16} \mathrm{~N}_{2} \mathrm{O}_{4}$ & 0.36 & $150-152$ \\
\hline $\mathbf{7 c}$ & $6-\mathrm{CH}_{3}$ & $4-\mathrm{F}-\mathrm{C}_{6} \mathrm{H}_{4}$ & $\mathrm{C}_{18} \mathrm{H}_{13} \mathrm{FN}_{2} \mathrm{O}_{3}$ & 0.39 & $168-170$ \\
\hline $\mathbf{7 d}$ & $6-\mathrm{CH}_{3}$ & $2-\mathrm{OH}-\mathrm{C}_{6} \mathrm{H}_{4}$ & $\mathrm{C}_{18} \mathrm{H}_{14} \mathrm{~N}_{2} \mathrm{O}_{4}$ & 0.48 & $230-232$ \\
\hline $\mathbf{7 e}$ & $6-\mathrm{CH}_{3}$ & $\mathrm{C}_{6} \mathrm{H}_{5}-\mathrm{CH}=\mathrm{CH}$ & $\mathrm{C}_{21} \mathrm{H}_{16} \mathrm{~N}_{2} \mathrm{O}_{4}$ & 0.32 & $226-228$ \\
\hline $\mathbf{7 f}$ & $6-\mathrm{CH}_{3}$ & $3,4,5-$ tri- $-\mathrm{OCH}_{3}-\mathrm{C}_{6} \mathrm{H}_{2}$ & $\mathrm{C}_{21} \mathrm{H}_{20} \mathrm{~N}_{2} \mathrm{O}_{6}$ & 0.57 & $208-210$ \\
\hline $\mathbf{7 g}$ & $6-\mathrm{CH}_{3}$ & $3,4-d i-\mathrm{OCH}_{3}-\mathrm{C}_{6} \mathrm{H}_{3}$ & $\mathrm{C}_{20} \mathrm{H}_{18} \mathrm{~N}_{2} \mathrm{O}_{5}$ & 0.43 & $148-150$ \\
\hline $\mathbf{7 h}$ & $6-\mathrm{CH}_{3}$ & $3-\mathrm{OCH} \mathrm{H}_{3}-\mathrm{C}_{6} \mathrm{H}_{4}$ & $\mathrm{C}_{19} \mathrm{H}_{16} \mathrm{~N}_{2} \mathrm{O}_{4}$ & 0.39 & $218-220$ \\
\hline $\mathbf{7 i}$ & $6-\mathrm{CH}_{3}$ & $3-\mathrm{Cl}_{2}-\mathrm{C}_{6} \mathrm{H}_{4}$ & $\mathrm{C}_{18} \mathrm{H}_{13} \mathrm{ClN}_{2} \mathrm{O}_{3}$ & 0.29 & $160-162$ \\
\hline $\mathbf{7 j}$ & $6-\mathrm{CH}_{3}$ & $2-\mathrm{Cl}_{2}-\mathrm{C}_{6} \mathrm{H}_{4}$ & $\mathrm{C}_{18} \mathrm{H}_{13} \mathrm{ClN}_{2} \mathrm{O}_{3}$ & 0.31 & $250-252$ \\
\hline $\mathbf{7 k}$ & $6-\mathrm{CH}_{3}$ & $4-\mathrm{Cl}_{-} \mathrm{C}_{6} \mathrm{H}_{4}$ & $\mathrm{C}_{18} \mathrm{H}_{14} \mathrm{ClN}_{2} \mathrm{O}_{3}$ & 0.36 & $230-232$ \\
\hline $\mathbf{7 l}$ & $6-\mathrm{CH}_{3}$ & $3-\mathrm{NO}_{2}-\mathrm{C}_{6} \mathrm{H}_{4}$ & $\mathrm{C}_{18} \mathrm{H}_{13} \mathrm{~N}_{3} \mathrm{O}_{5}$ & 0.42 & $212-214$ \\
\hline
\end{tabular}




\section{BIOLOGICAL ACTIVITY}

The present work deals with the antimicrobial screening of the compounds (7a-1). The minimum inhibition concentration (MIC) values were determined in the present study.

\section{1. Preparation of Muller Hinton agar plates with antibacterial test agents:}

$10 \mathrm{mg}$ of compound was dissolved in $2 \mathrm{ml}$ of DMSO to prepare stock solution of the compound to be tested. $1 \mathrm{ml}$ of this stock was added to $19 \mathrm{ml}$ of molten Muller Hinton agar medium and poured in a sterile empty plate, allowed it to solidify. It gave the final concentration of the compound $1000 \mathrm{mg} / \mathrm{ml}$ in the agar medium. The stock solution was further diluted by two fold dilution procedure to obtain the desired concentration of the compound in the agar medium plate. i.e. $1000 \mathrm{mg} / \mathrm{ml}, 500 \mathrm{mg} / \mathrm{ml}, 250 \mathrm{mg} / \mathrm{ml}, 125 \mathrm{mg} / \mathrm{ml}, 75$ $\mathrm{mg} / \mathrm{ml}, 37 \mathrm{mg} / \mathrm{ml}$ and $18 \mathrm{mg} / \mathrm{ml}$.

\section{2. Incubation of Microbial Cultures:}

$5 \mathrm{ml}$ of Muller Hinton broth media were inoculated with the needed microbial cultures and kept for 2 to $6 \mathrm{hrs}$ for incubation at $37{ }^{\circ} \mathrm{C}$ temperature. After getting proper growth the culture solution were read against sterile Muller Hinton broth blank, using 0.5 Mc farlan standards at $620 \mathrm{~nm}$ wavelength in spectrophotometer. Appropriate dilution of microbial culture was made to match using $0.5 \mathrm{Mc}$ farlan standards. The final dilution of microbial cultures contains 104 cells $/ \mathrm{ml}$. $2 \mathrm{ml}$ of this diluted microbial culture were used to spot inoculate the plates.

\section{3. The plates with antimicrobial test agents:}

\section{a) Controls:}

Solvent control was kept adding $1 \mathrm{ml}$ of DMSO only to check the inhibitory effect on microbes. Organism control was kept without adding compound.

\section{b) Evaluation:}

The plates were kept at $37{ }^{\circ} \mathrm{C}$ temperature for $24 \mathrm{hrs}$ to get visible growth of microorganisms. Plates were check for growth. Microbes grown in big colony if not inhibited, grown in small size colonies or scanty growth if inhibited, no growth was found in case when they were killed by cidal effect of synthetic compound. Various organisms used in study of antibacterial activity are:

\section{3. 1. Gram positive}

1. S. aureus

2. B. subtilis

\section{3. 2. Gram negative}

1. E. coli,

2. P. aeruginosa,

3. K. pneunonae,

4. Enterobacter 
Table 2

\begin{tabular}{|c|c|c|c|c|c|c|}
\hline \multirow{3}{*}{ Code } & \multicolumn{6}{|c|}{ Micro organism (MIC in $\mu \mathrm{gm} / \mathrm{ml}$ ) } \\
\hline & \multicolumn{4}{|c|}{ Gram negative } & \multicolumn{2}{|c|}{ Gram positive } \\
\hline & $\begin{array}{c}\text { Escherichia } \\
\text { coli }\end{array}$ & Enterabacter & $\begin{array}{c}P . \\
\text { aeruginosa }\end{array}$ & $\begin{array}{c}\text { K. } \\
\text { pneumonae }\end{array}$ & S. aureus & $\begin{array}{c}\text { Bacilluas } \\
\text { subtilis }\end{array}$ \\
\hline $7 a$ & $<18$ & $<18$ & - & $<18$ & $<18$ & $<18$ \\
\hline $7 b$ & - & - & $<250$ & - & $<125$ & - \\
\hline 7c & $<37$ & - & $<37$ & $<37$ & $<37$ & $<37$ \\
\hline $7 d$ & $<125$ & $<125$ & - & $<125$ & - & - \\
\hline $7 e$ & $<250$ & $<250$ & - & $<250$ & - & - \\
\hline $7 f$ & $<75$ & $<75$ & - & $<75$ & - & - \\
\hline $7 g$ & - & - & $<250$ & - & - & - \\
\hline $7 \mathrm{~h}$ & - & - & $<75$ & - & - & - \\
\hline $7 \mathbf{i}$ & - & - & $<75$ & $<75$ & $<75$ & $<75$ \\
\hline $7 \mathbf{j}$ & - & - & - & - & $<75$ & - \\
\hline $7 k$ & - & - & - & $<18$ & $<18$ & $<18$ \\
\hline 71 & $<75$ & - & $<125$ & - & - & - \\
\hline
\end{tabular}

\section{RESULT AND DISSCUTION}

The constitutions of newly synthesized compounds were supported by IR, ${ }^{1} \mathrm{H}$ NMR, Mass and ${ }^{13} \mathrm{C}$ NMR spectral study. Newly synthesized $2 \mathrm{H}$-chromene-4-carbohydrazide, the carbonyl $(>\mathrm{C}=\mathrm{O})$ for the ring and the ester stretching of side chain is observed at range of $\sim 1720-1700 \mathrm{~cm}^{-1}$ and $1740-1710 \mathrm{~cm}^{-1}$ respectively. The confirmation of methyl group in the ring shows the $\mathrm{CH}_{3}$ bending in range of $\sim 1385-1360 \mathrm{~cm}^{-1}$ and $2854-2823 \mathrm{~cm}^{-1}$ (7a). A sharp band of -NH stretch is observed at $3423 \mathrm{~cm}^{-1}$.

The sharp carbonyl band $(>\mathrm{C}=\mathrm{O})$ is seen at $1718 \mathrm{~cm}^{-1}$ which indicates the presence of carbonyl $(>\mathrm{C}=\mathrm{O})$ group in the ring. The $(>\mathrm{C}=\mathrm{O})$ for the amide carbonyl bending is observed at $1654 \mathrm{~cm}^{-1}$. In ${ }^{1} \mathrm{H}$ NMR Spectrum of (7a) presence of methylene protons $-\mathrm{CH}_{2}$ is confirmed by observing a singlet in up field at $3.52 \delta \mathrm{ppm}$, another singlet at $2.26 \delta \mathrm{ppm}$, indicates the presence of $\mathrm{C}_{3}$ proton of coumarin ring.

The protons of the substituted phenyl ring give multiplet at 7.39-7.46 $\delta \mathrm{ppm}(\mathrm{m}, 3 \mathrm{H})$, 7.78-7.91 $\delta \mathrm{ppm}(\mathrm{m}, 3 \mathrm{H})$, The results of antimicrobial activity (Table 2) shows that compound 7a, 7c, 7k, showed excellent activity against $K$. pneumonae, S. aureus, Bacilluas subtilis, while compound 7f, 71 maximum activity against Escherichia coli. Other compounds not show inhibition in bacterial culture. 


\section{CONCLUSION}

The method used for preparation procedure is an efficient, simple, rapid and ecofriendly and method for preparation of N'-benzylidene-2-(6-methyl-2-oxo-2H-chromen-4-yl) acetohydrazide derivatives by the reaction of chromen-4-yl acetohydrazide and various substituted benzaldehyde. Antibacterial screening of these all synthesized compound was carried out. The compound containing the halogen substituent shows greater antimicrobial activity.

\section{ACKNOWLEDGEMENTS}

The authors are thankful to Department of Chemistry, Saurashtra University, Rajkot and specially indebted to "National Facility for Drug Discovery through New Chemical Entities (NCE's), Development \& Instrumentation Support to Small Manufacturing Pharma Enterprises”, a programme under Drug \& Pharma Research Support (DPRS) jointly funded by Department of Science \& Technology, New Delhi, Government of Gujarat (Industries Commissionerate) \& Saurashtra University, Rajkot.

\section{References}

[1] Manolov, I.; Danchev, N. D., Eur. J. Med. Chem. Chim. Ther. 30 (1995) 531-536.

[2] Shaker, R.M., Pharmazie 51 (1996) 148.

[3] Nofal, Z.M.; El-Zahar, M.; Abd El-Karim, S., Molecules 5 (2000) 99-113.

[4] Vithal B. Jadhav, Susanta K. Nayak, T.N. Guru Row, M.V. Kulkarni, European Journal of Medicinal Chemistry 45 (2010) 3575-3580.

[5] Maxwell, A., Trends Microbiol. 5 (1997) 102.

[6] Calia, H.; Hoerman, L.; Schultz, P.; Lebeau, L.; Mallouh, V.; Wigley, D. B.; Wang, J.C.; Mioskowski, C.; Oudet, P., J. Mol. Biol. 236 (1994) 618.

[7] Boehm H. J., et al., J. Med. Chem. 43 (2000) 664.

[8] Shiozawa et al., Proc. Am. Assoc. Cancer Res. 2002, 43, 2460.

[9] Nakatomi, et al., Jpn. J. Cancer Res. 92 (2001) 1973.

[10] Kalpesh S., Parikhand Sandip, P. Vyas, Archives of Applied Science Research 4 (2012) 1578-1580.

[11] Mostafa M. H. Khalil, Eman H. Ismail, Gehad G. Mohamed, Ehab M. Zayed, Ahmed Badr, Open Journal of Inorganic Chemistry 2 (2012) 13-21.

[12] Robert Southgate, Contemp. Org. Synth. 1 (1994) 417-431.

[13] Stefan France, Anthony Weatherwax, Andrew E. Taggi, Thomas Lectka, Acc. Chem. Res. 37(8) (2004) 592-600.

[14] Santosh Kumar, Niranjan M. S., Chaluvaraju K. C., Jamakhandi C. M, Dayanand Kadadevar, Journal of Current Pharmaceutical Research 1 (2010) 39-42.

[15] Amel F. El Husseiny, Elham S.Aazam, Jemila Al Shehary, In organic chemistry an Indian Journal 3 (2008) 64-68. 
[16] V. S. V. Satyanarayana, P. Sreevani, Amaravadi Sivakumar, V. Vijayakumar, ARKIVOC xvii (2008) 221-233.

[17] T. N. Mohammed Musthafa, Zeba N. Siddiqui, Fohad N. Husain, Iqbal Ahmad; Med Chem Res 20 (2011) 1473-1481.

[18] Omaima M. Abdelhafez, Kamelia M. Amin, Hamed I. Ali, Mohamed M. Abdalla, Rasha Z. Batran, J. Med. Chem. 55 (2012) 10424-10436.

[19] Wang P-H., Keck J. G., Lien E. J., Lai M. M. C., J. Med. Chem. 33 (1990) 608.

[20] Das A.; Trousdale M. D.; Ren S.; Lien E.; J. Antiviral Res. 44 (1999) 201-208.

[21] Pathak P., Jolly V. S., Sharma K. P., Orient. J. Chem. 16 (2000) 161-162.

[22] Kuz'min V. E., et. al., Acta Biochim. Polon. 47 (2000) 867-876.

[23] Aamer Saeed, Muhammad Siraj Khan, Hummera Rafique, Mohammad Shahid, Jamshed Iqbal, Recent Bioorganic Chemistry Articles Bioorganic Chemistry 52 (2014) 1-7.

[24] Jason Wagner, Balendu Sankara Avvaru, Arthur H. Robbins, Andrea Scozzafava, Claudiu T. Supuran, Robert McKenna, Bioorg Med Chem. 18 (2010) 4873-4878.

[25] Katharigatta Narayanaswamy Venugopala, et al., European Journal of Medicinal Chemistry 65 (2013) 295-303.

[26] Rakesh B. Pate, Kishor H. Chikhalia, Christophe Pannecouque, Erik de Clercq, J. Braz. Chem. Soc. 18(2) (2007). 